\title{
An Adaptive-Scaling Algorithm for OFDM PAR Reduction Using Active Constellation Extension
}

\author{
Luqing Wang and Chintha Tellambura \\ Department of Electrical and Computer Engineering \\ University of Alberta \\ Edmonton, Alberta T6G 2V4 Canada \\ Ph: 17804927228 Fax: 17804921811 \\ Email: \{wlq, chintha\}@ece.ualberta.ca
}

\begin{abstract}
The Active Constellation Extension (ACE) technique is a lossless (in terms of throughput) Peak-to-Average Power Ratio (PAR) reduction technique that adaptively extends the signal constellation while ensuring that the minimum distance between any two constellation points does not decrease. However, this technique increases the average transmit power. In this paper, we propose an adaptive-scaling algorithm for the implementation of ACE. This algorithm, based on the clipping and filtering technique, uses only the peak samples of clipping noise to reduce PAR. Simulation results show that the proposed algorithm has better PAR reduction, lower complexity and smaller BER than the previously proposed Smart Gradient-Project ACE algorithm.
\end{abstract}

\section{INTRODUCTION}

A main drawback of Orthogonal Frequency Division Multiplexing (OFDM) systems is the high Peak-to-Average Power Ratio (PAR) [1]. Large PAR requires a linear High Power Amplifier (HPA), which, however, is not efficiently used. If the linear range of HPA is not sufficient, large PAR leads to in-band distortion and out-of-band radiation [1]. Various PAR reduction techniques have been proposed, including multiple signal representation [2]-[4], coding [5], and peak cancelation techniques [6]-[14].

Multiple signal representation techniques such as Selected Mapping (SLM) and Partial Transmit Sequences (PTS) are suitable for OFDM systems with a large number of subcarriers [2]-[4]. However, these techniques may require side information at the receiver to decode the input symbols, and incorrectly received side information results in burst errors. Although coding techniques such as block codes, convolutional codes, and Golay complementary sequences may also be used to reduce PAR [5], these techniques have low code rates when the number of subcarriers increases.

Peak cancelation techniques use a band-limited Peak Cancelation Signal (PCS) to cancel large peaks in OFDM signals. Depending on the spectrum constraints on the PCS, these techniques can be divided into several groups. Allowing the PCS to freely distribute over the whole OFDM frequency band gives rise to the clipping and filtering approach which clips the signal and eliminates the out-of-band radiation by filtering [6][10]. Since the in-band clipping noise cannot be eliminated, this approach leads to increased bit error rate (BER). The tone reservation technique [11]-[13] reserves a small number of unused subcarriers to reduce the PAR. Since the PCS is orthogonal to data symbols, this technique does not increase the BER with the cost of increased transmission power and loss of throughput.

The Active Constellation Extension (ACE) technique [14] reduces the PAR without any loss of throughput. It adaptively extends the signal constellation so that the distance between any two constellation points does not decrease. In [14], ACE is implemented via a Smart Gradient-Project (SGP) algorithm. ACE however increases the average power of the OFDM signal, precipitating BER increase compared to conventional OFDM.

This paper proposes an adaptive-scaling algorithm for the implementation of ACE. This algorithm is based on clipping and filtering, and uses only the peak samples of clipping noise to reduce PAR maximally. Simulation results show that our proposed algorithm outperforms SGP in terms of PAR reduction, BER, and complexity. The proposed algorithm can also be applied with the tone reservation technique.

This paper is organized as follows: Section II characterizes the OFDM System and gives a brief review of ACE. The adaptive-scaling algorithm is then proposed in Section III. In Section IV, the proposed algorithm is compared with SGP by simulation. The conclusion is given in Section V.

\section{OFDM AND ACtive Constellation Extension TECHNIQUE}

\section{A. Characterization of OFDM System}

In OFDM systems, the time domain signal $x(t)$ may be written as

$$
x(t)=\frac{1}{\sqrt{N}} \sum_{k=-\frac{N}{2}}^{\frac{N}{2}-1} X_{k} e^{j 2 \pi k t / T}, \quad 0 \leq t \leq T,
$$

where $\mathbf{X}=\left[X_{-\frac{N}{2}}, X_{-\frac{N}{2}+1}, \ldots, X_{\frac{N}{2}-1}\right]$ represents the input OFDM symbol, $N^{2}$ is the number of subcarriers, and $T$ is the OFDM symbol period. The PAR of $x(t)$ can be defined as

$$
\xi=\frac{\max _{t \in[0, T)}|x(t)|^{2}}{P_{a v}}
$$


where $P_{a v}=E\left\{|x(t)|^{2}\right\}=E\left\{\left|X_{k}\right|^{2}\right\}$ is the average power. Note that $\xi$ can be very large. For example, when PSK symbols are used, $\xi$ can be as large as $N$. However, since such a large PAR occurs rarely, it is more useful to consider $\xi$ as a random variable and use a statistical description given by the Complementary Cumulative Distribution Function (CCDF), defined as the probability that $\mathrm{PAR}$ exceeds $\xi_{0}$, i.e., $\operatorname{Pr}[\xi>$ $\left.\xi_{0}\right]=P_{c}$.

To approximate $\xi$ in discrete-time domain $x(t)$ is sampled to obtain $x_{n}$ as

$$
x_{n}=\frac{1}{\sqrt{N}} \sum_{k=-\frac{N}{2}}^{\frac{N}{2}-1} X_{k} e^{j 2 \pi \frac{n k}{J N}}, \quad n=0, \ldots, J N-1,
$$

where $J$ is the oversampling factor. The discrete-time PAR can be defined similar to (2), which approaches $\xi$ when $J \geq 4 . x_{n}$ can be calculated by applying a $(J N)$-point Inverse Discrete Fourier Transform (IDFT) on the extended input vector $\mathbf{X}_{\mathrm{ext}}$,

$$
\mathbf{X}_{\mathrm{ext}}=[X_{0}, \ldots, X_{\frac{N}{2}-1}, \underbrace{0, \ldots, 0}_{(J-1) N \text { zeros }}, X_{-\frac{N}{2}}, \ldots, X_{-1}] .
$$

\section{B. Active Constellation Extension Technique}

A signal constellation is a set of points on the complex plane. Data symbols $X_{k}$ are drawn from a given constellation usually with equal probability. The minimum Euclidean distance $d_{\min }$ of a constellation determines its BER performance.

ACE [14] reduces PAR by extending the outer constellation points with a constraint that the minimum distance between any two points does not decrease. As an example, Fig. 1 shows the feasible extension region (shaded area) for the QPSK constellation, where circles represent the original constellation points. Let the modified OFDM symbol be

$$
\hat{X}_{k}=X_{k}+C_{k}, \quad k=0,1, \ldots, N-1,
$$

where $\mathbf{C}=\left[C_{0}, C_{1}, \ldots, C_{N-1}\right]$ is the extension vector. For QPSK constellation, $C_{k}$ must satisfy

$$
\begin{aligned}
& \mathfrak{R e}\left[C_{k}\right] \mathfrak{R e}\left[X_{k}\right] \leq 0, \text { and } \\
& \mathfrak{I m}\left[C_{k}\right] \mathfrak{I m}\left[X_{k}\right] \leq 0,
\end{aligned}
$$

where $\mathfrak{R e}[x]$ and $\mathfrak{I m}[x]$ are the real and imaginary parts of $x$, respectively. In order to prevent the appearance of large $C_{k}$ [12], the PAR is redefined as ${ }^{1}$

$$
\xi=\frac{\max \left|x_{n}+c_{n}\right|^{2}}{E\left\{\left|x_{n}\right|^{2}\right\}},
$$

where $c_{n}=\operatorname{IDFT}\left\{\mathbf{C}_{\text {ext }}\right\}$, and $\mathbf{C}_{\text {ext }}$ is obtained from $C_{k}$ in the same fashion of (4).

Minimizing $\xi$ is equivalent to

$$
\begin{array}{ll}
\min _{\mathbf{C}} E & \\
& \text { subject to: } E \geq 0, \\
& \left|x_{n}+c_{n}\right|^{2} \leq E, \\
& \mathfrak{R e}\left[C_{k}\right] \mathfrak{R e}\left[X_{k}\right] \leq 0, \text { and } \\
& \mathfrak{I m}\left[C_{k}\right] \mathfrak{I m}\left[X_{k}\right] \leq 0,
\end{array}
$$

\footnotetext{
${ }^{1}$ This definition is implicitly used in the simulations of [14].
}

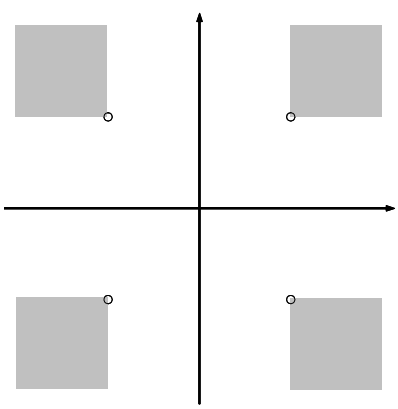

Fig. 1. Shaded area shows the feasible extension region for the QPSK constellation, where circles represent original QPSK constellation points.

which is a Quadratically Constrained Quadratic Program (QCQP).

\section{Clipping-based Algorithm}

In [14], clipping and filtering is used to reduce the PAR. In each iteration, the time domain OFDM signal $x_{n}$ is first clipped by a Soft Limiter (SL) [12]. The clipped OFDM signal $\tilde{x}_{n}$ is

$$
\tilde{x}_{n}= \begin{cases}A e^{j \phi_{n}}, & \left|x_{n}\right|>A, \\ x_{n}, & \left|x_{n}\right| \leq A,\end{cases}
$$

where $A$ is the predefined threshold and $\phi_{n}$ is the phase of $x_{n}$. The clipping noise

$$
f_{n}=x_{n}-\tilde{x}_{n}
$$

is converted to the frequency domain by a Discrete Fourier Transform (DFT) operation to obtain $F_{k}$. The out-of-band terms of $F_{k}$ are set to zero and the in-band terms are adjusted to obtain $\hat{F}_{k}$. For the QPSK constellation, $F_{k}$ must satisfy (5) with $C_{k}$ replaced by $F_{k}$. In general, the principle of this adjustment is that, if a resulted data symbol $\left(X_{k}-F_{k}\right)$ is out of its feasible extension region, $F_{k}$ is adjusted to $\hat{F}_{k}$ such that $\left(X_{k}-\hat{F}_{k}\right)$ is the nearest point of $\left(X_{k}-F_{k}\right)$ in the feasible extension region. Note that a data symbol is only allowed to move away from its original constellation point for the sake of simplifying the algorithm. An IDFT is applied to $\hat{F}_{k}$ to obtain the modified clipping noise $\hat{f}_{n}$. Note that, at this stage, the difference between ACE and the clipping and filtering technique is that the latter only set the out-of-band terms of $F_{k}$ to zero and does not modify any in-band terms.

The clipping-noise term $\hat{f}_{n}$ is then scaled by a factor $\beta$ and subtracted from $x_{n}$ to obtain the PAR-reduced OFDM signal as follows:

$$
\hat{x}_{n}=x_{n}-\beta \hat{f}_{n} .
$$

That is,

$$
c_{n}=-\beta \hat{f}_{n} .
$$

In [14], an SGP algorithm is proposed to find

$$
\beta^{*}=\min _{\beta_{n}} \frac{\left|x_{n_{\max }}\right|-\left|x_{n}\right|}{\hat{f}_{n}^{\text {proj }}-\hat{f}_{n_{\max }}^{\text {proj }}}
$$

where

$$
n_{\max }=\arg \max _{n}\left|x_{n}\right|,
$$


and

$$
\hat{f}_{n}^{\text {proj }}=\frac{\mathfrak{R e}\left[x_{n} \hat{f}_{n}^{*}\right]}{\left|x_{n}\right|},
$$

where $(\cdot)^{*}$ is the complex conjugate.

While ACE does not decrease $d_{\min }$, the average power of OFDM symbols is increased, which results in an increased BER compared to conventional OFDM. However, this BER increase can be exploited to gain further reduction of PAR. In the next section, we will analyze ACE, and propose an adaptive-scaling algorithm, which will improve PAR reduction and BER degradation associated with conventional ACE.

\section{An AdAptive-ScAling ACE Algorithm}

\section{A. Find the optimal $\beta$}

We first calculate the optimal $\beta$, denoted as $\beta^{(\mathrm{opt})}$. Note that ACE can be viewed as a special case of the tone reservation technique [12], where all subcarriers are used for data transmission, and the reserved subcarriers are adaptively selected in each iteration such that the data symbols can only be extended in their feasible extension regions. Therefore, we can apply the analysis results of tone reservation [11] to ACE.

While clipping $x_{n}$ using an SL, $f_{n}$ can be represented as a series of pulses unless $A$ is small,

$$
f_{n}=\sum_{i} f_{i, n}
$$

where $f_{i, n}$ is the $i$-th clipping pulse which can be approximated as a parabolic arc with pulse duration $\tau_{i}$ [11]. On the other hand,

$$
\hat{f}_{n}=f_{n} \star h_{n}=\frac{1}{\sqrt{N}} \sum_{k=-\frac{J N}{2}}^{\frac{J N}{2}-1} F_{k} H_{k} e^{j 2 \pi \frac{n k}{J N}},
$$

where $\star$ represents linear convolution, $F_{k}$ is the DFT of $f_{n}$, and $h_{n}$ is an ideal multiple-passband filter with the (discrete) frequency response as

$$
H_{k}= \begin{cases}1, & k \in \mathcal{R}, \\ 0, & \text { otherwise },\end{cases}
$$

with $\mathcal{R}$ representing the indexes of the subcarriers that satisfy the ACE criteria. Note that smaller sidelobes of $h_{n}$ lead to larger PAR reduction [11]. The PAR reduced signal $\hat{x}_{n}$ is

$$
\hat{x}_{n}=x_{n}-\beta \hat{f}_{n}=A e^{j \phi_{n}}+f_{n}-\beta \hat{f}_{n} .
$$

It can be shown [11] that $\hat{f}_{n}$ is roughly a constant in the time duration of any large pulse of $f_{n}$ unless $\beta$ is very large ${ }^{2}$. Therefore, if we clip $\hat{x}_{n}$ using the same SL, the clipping noise $f_{n}^{(1)}$ is also a series of parabolic pulses, which consist of some "newly generated" pulses that do not exist in $f_{n}$, and pulses that shrink from $f_{n}$.

The power of $f_{n}^{(1)}$ is the out-of-range power of $\hat{x}_{n}$, which must be minimized by optimizing $\beta$. Therefore, the objective function is

$$
\min _{\beta} P
$$

\footnotetext{
${ }^{2}$ When $\beta$ is very large, the PAR is usually increased.
}

where

$$
\begin{aligned}
P & =\sum_{n}\left|f_{n}^{(1)}\right|^{2}=\sum_{\left|\hat{x}_{n}\right|>A}\left(\left|\hat{x}_{n}\right|-A\right)^{2} \\
& =\sum_{n \in \mathcal{S}_{1}}\left(\left|\hat{x}_{n}\right|-A\right)^{2}-\sum_{\substack{n \in \mathcal{S}_{1} \\
\left|\hat{x}_{n}\right| \leq A}}\left(\left|\hat{x}_{n}\right|-A\right)^{2}+\sum_{n \in \mathcal{S}_{2}}\left(\left|\hat{x}_{n}\right|-A\right)^{2},
\end{aligned}
$$

with $\mathcal{S}_{1}=\left\{n:\left|f_{n}\right|>0\right\}$ representing the index set of all clipping pulses, and $\mathcal{S}_{2}=\left\{n:\left|f_{n}\right|=0\right.$ and $\left.\left|\hat{x}_{n}\right|>A\right\}$. Since $f_{n}^{(1)}$ is a series of parabolic pulses, minimizing $P$ is equivalent to minimizing the peaks of $f_{n}^{(1)}$, i.e., minimizing

$$
\hat{P}=\underbrace{\sum_{n \in \mathcal{S}_{p}}\left(\left|\hat{x}_{n}\right|-A\right)^{2}}_{P_{1}}-\underbrace{\sum_{\substack{n \in \mathcal{S}_{p} \\\left|\hat{x}_{n}\right| \leq A}}\left(\left|\hat{x}_{n}\right|-A\right)^{2}}_{P_{2}}+\underbrace{\sum_{n \in \mathcal{S}_{p}^{+}}\left(\left|\hat{x}_{n}\right|-A\right)^{2}}_{P_{3}},
$$

where $\mathcal{S}_{p}=\left\{n: n \in \mathcal{S}_{1},\left|x_{n}\right|>\left|x_{n-1}\right|\right.$ and $\left.\left|x_{n}\right| \geq\left|x_{n+1}\right|\right\}$ is the index set of the peaks of $f_{n}$, and $\mathcal{S}_{p}^{+}=\{n: n \in$ $\mathcal{S}_{2},\left|\hat{x}_{n}\right|>\left|\hat{x}_{n-1}\right|$ and $\left.\left|\hat{x}_{n}\right| \geq\left|\hat{x}_{n+1}\right|\right\}$ is the index set of the peaks of "newly generated" pulses whose amplitudes are larger than $A$.

Eqn. (14) implies that $\beta^{\text {(opt) }}$ must both minimize the peaks of $x_{n}$ and prevent any large "newly generated" pulses, which in turn implies that $\beta^{(\mathrm{opt})}$ could not be large. Taking this fact into account, $P_{2}$ and $P_{3}$ are small and their difference can be omitted. Therefore,

$$
\begin{aligned}
\hat{P} & \approx P_{1} \\
& =\sum_{n \in \mathcal{S}_{p}}\left|\hat{x}_{n}-A e^{j \hat{\phi}_{n}}\right|^{2} \\
& =\sum_{n \in \mathcal{S}_{p}}\left|f_{n}-\beta \hat{f}_{n}+A\left(e^{j \phi_{n}}-e^{j \hat{\phi}_{n}}\right)\right|^{2},
\end{aligned}
$$

where $\hat{\phi}_{n}$ is the phase of $\hat{x}_{n}$.

Since $\beta^{\text {(opt) }}$ is not very large, we can see that most probably $\left|x_{n}\right|=\left|A e^{j \phi_{n}}+f_{n}\right| \gg\left|\beta \hat{f}_{n}\right|$, i.e., $\beta \hat{f}_{n}$ could not significantly change the phase of $x_{n}$. Therefore, $\hat{\phi}_{n} \approx \phi_{n}$ and

$$
\hat{P} \approx \sum_{n \in \mathcal{S}_{p}}\left|f_{n}-\beta \hat{f}_{n}\right|^{2} .
$$

The optimal solution is

$$
\beta^{(\mathrm{opt})}=\frac{\mathfrak{R e}\left[\sum_{n \in \mathcal{S}_{p}} f_{n} \hat{f}_{n}^{*}\right]}{\sum_{n \in \mathcal{S}_{p}}\left|\hat{f}_{n}\right|^{2}} .
$$

\section{B. Reduce Average Power Increase of the OFDM Signal}

The ACE technique becomes the clipping and filtering technique if we drop off the constraints on $C_{k}$ (e.g. (5) for QPSK) and distribute the clipping noise into all subcarriers. In this case, $\hat{x}_{n}$ can be written as

$$
\hat{x}_{n}=\alpha x_{n}+d_{n},
$$

where $d_{n}$ is the zero-mean distortion term uncorrelated to $x_{n}$, and $\alpha<1$ indicates a decrease of the average power of $x_{n}$. 
Therefore, we can use the clipping and filtering technique to counteract the power increase caused by the ACE technique.

Distributing the clipping noise into all subcarriers implies $H_{k}=1$ for all $k$, which gives the largest PAR reduction [11]. In other words, a small decrease of average power may lead to large PAR reduction. Moreover, since the power spectrum of $\hat{f}_{n}$ is white when using the clipping and filtering technique, the inband distortion is equally distributed into all subcarriers, meaning that no subcarrier is severely distorted and $d_{\min }$ is not greatly decreased. Therefore, combining clipping and filtering with the ACE technique may give rise to large PAR reduction and small BER increase.

The new algorithm is now summarized as follows.

Algorithm 1 (Adaptive-Scaling Algorithm):

For each OFDM symbol,

1) Apply the ACE technique:

a) Clip $x_{n}$ using an SL and obtain $f_{n}$.

b) Obtain $\hat{f}_{n}$ by modifying $f_{n}$ in the frequency domain to satisfy (5).

c) Calculate $\beta^{\text {(opt) }}$ using (17).

d) Find $\hat{x}_{n}$ using (8).

2) Calculate the average power $\hat{P}_{\text {av }}$ of $\hat{x}_{n}$.

3) If $\hat{P}_{\mathrm{av}} \leq P_{\mathrm{av}}$, where $P_{\mathrm{av}}$ is the average power of the conventional OFDM signal, then apply the ACE technique (c.f. step 1); otherwise, apply the clipping and filtering technique, i.e.,

a) Clip $x_{n}$ using an SL and obtain $f_{n}$.

b) Obtain $\hat{f}_{n}$ by filtering out the out-of-band terms in $f_{n}$.

c) Calculate $\beta^{\text {(opt) }}$ using (17).

d) Find $\hat{x}_{n}$ using (8).

4) If $\max _{n}\left|\hat{x}_{n}\right|>A$ and the iteration number is less than, say, $L$, then go to Step 2; otherwise, stop the algorithm and output $\hat{x}_{n}$.

\section{Complexity Comparison}

The adaptive-scaling algorithm has smaller complexity than the SGP algorithm in the calculation of $\beta$. In (9), calculating $\beta^{*}$ involves all samples of $\hat{f}_{n}$, which requires $2(J N-1)$ complex additions, $3 J N$ complex multiplications/divisions, and $J N-1$ comparisons.

In contrary, (17) only involves the peaks of $f_{n}$. Denoting the number of peaks of $f_{n}$ as $N_{p}$ and denoting the number of samples of $f_{n}$ as $N_{f}$, calculating (17) requires $N_{f}-1$ comparisons (to find all peaks of $f_{n}$ ), $3 N_{p}$ complex multiplications/divisions, and $2\left(N_{p}-1\right)$ complex additions. $N_{f}$ and $N_{p}$ are random variables. However, their means can be calculated as follows [11].

$$
\begin{aligned}
& \bar{N}_{f}=J N e^{-A^{2} /\left(2 \sigma^{2}\right)}, \\
& \bar{N}_{p}=N \sqrt{\frac{\pi}{6}} \frac{A}{\sigma} e^{-A^{2} /\left(2 \sigma^{2}\right)},
\end{aligned}
$$

where $\sigma$ is the variance of $x_{n}$. For example, when $N=256$, $J=4, A=6 \mathrm{~dB}$, and $\sigma=\frac{1}{\sqrt{2}}$, we have $\bar{N}_{f}=19.1136$ and $N_{p}=9.7566$. Therefore, (17) has much less complexity than (9).

\section{Simulation Results}

In this section, we compare the adaptive-scaling algorithm with SGP by simulation. We choose our simulation parameters to be consistent with those in [14], i.e., An $N=256$ OFDM system with uniformly distributed unitary QPSK symbols (i.e., $\left|X_{k}\right|=1$ ) as the input. $J=4$ for PAR reduction and $J=8$ for the final PAR measurement. The PAR reduction algorithm is applied when the PAR of an OFDM signal is greater than $6 \mathrm{~dB}$. The clipping level is set to $A=4.86 \mathrm{~dB}$. For both algorithms, the iteration number is $L=3$. When calculating the BER, each PAR reduced OFDM signal is scaled so that the average power is normalized to 1 before passing through the HPA.

Fig. 2 compares the adaptive-scaling algorithm and SGP. The PAR of original OFDM is also plotted in this figure as the dash curve. At PAR CCDF of $10^{-4}$, the SGP algorithm reduces the PAR to $7.6 \mathrm{~dB}$. On the other hand, the adaptivescaling algorithm reduces the PAR to $5.3 \mathrm{~dB}^{3}$. Therefore, the adaptive-scaling algorithm outperforms SGP by $2.3 \mathrm{~dB}$.

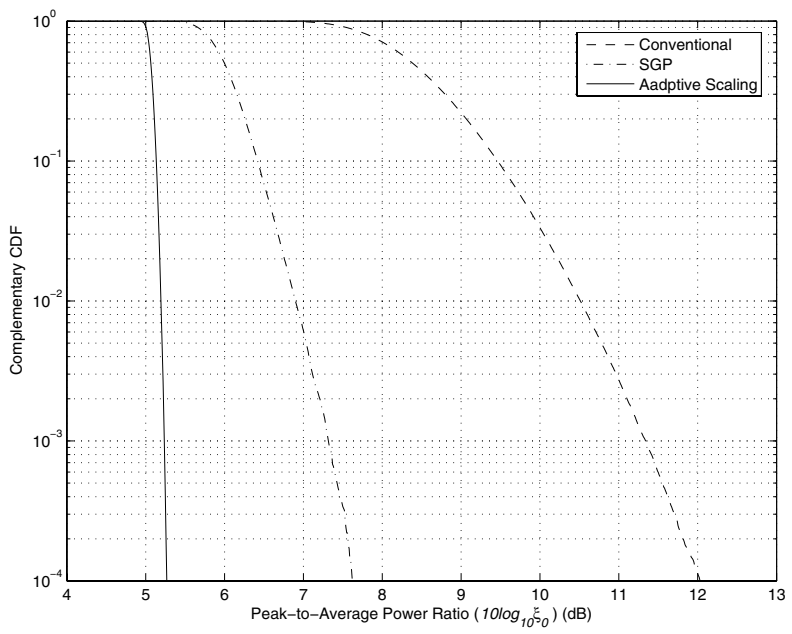

Fig. 2. PAR CCDF comparison of the adaptive-scaling algorithm and SGP, where the iteration number is $L=3$ for both algorithms.

The average computation time and the average power increase of these algorithms are listed in Table I, which is obtained on a Pentium IV 2.8G computer using Matlab 7 Service Pack 2. SGP increases the average power by $0.91 \mathrm{~dB}$; but the adaptive-scaling algorithm only increases the average power by $0.22 \mathrm{~dB}$. The computation time of the adaptivescaling algorithm is only $87 \%$ of that of SGP.

TABLE I

Average Computation time and Average Power Increase of the ADAPTIVE-SCALING ALGORITHM AND SGP

\begin{tabular}{|l|l|l|}
\hline & $\begin{array}{l}\text { Computation Time } \\
\text { (millisecond) }\end{array}$ & $\begin{array}{l}\text { Average Power Increase } \\
(\mathrm{dB})\end{array}$ \\
\hline Adaptive-Scaling & 4.89 & 0.22 \\
\hline SGP & 5.61 & 0.91 \\
\hline
\end{tabular}

${ }^{3}$ Those OFDM signals with PAR between $5.3 \mathrm{~dB}$ and $6 \mathrm{~dB}$ are not shown in Fig. 2 because their probability is very small. 
Now, we compare both algorithms in accordance with the OFDM signal power spectrum and BER by passing OFDM signals through a Solid State Power Amplifier (SSPA) with the input/output relationship as [1]

$$
y(t)=\frac{|x(t)|}{\left(1+\left(\frac{|x(t)|}{C}\right)^{2 p}\right)^{\frac{1}{2 p}}} e^{j \phi(t)},
$$

where $x(t)=|x(t)| e^{j \phi(t)}$ is the input, $y(t)$ is the output of the SSPA, and $C$ is the clipping threshold. The SSPA becomes the SL when $p \rightarrow \infty$. Usually, $p=3$ for practical SSPA. In the following simulations, $C=4.86 \mathrm{~dB}$.

Fig. 3 shows the power spectrum of both algorithms. As a comparison, the power spectrum of conventional OFDM (i.e., no PAR reduction technique is applied) over the SSPA is also shown in this figure as the dashed curve. Without any PAR reduction, the out-of-band radiation of the conventional OFDM signal is about $-25 \mathrm{~dB}$. While the SGP algorithm reduces outof-band radiation to $-32 \mathrm{~dB}$, the proposed adaptive-scaling algorithm entirely eliminates out-of-band radiation.

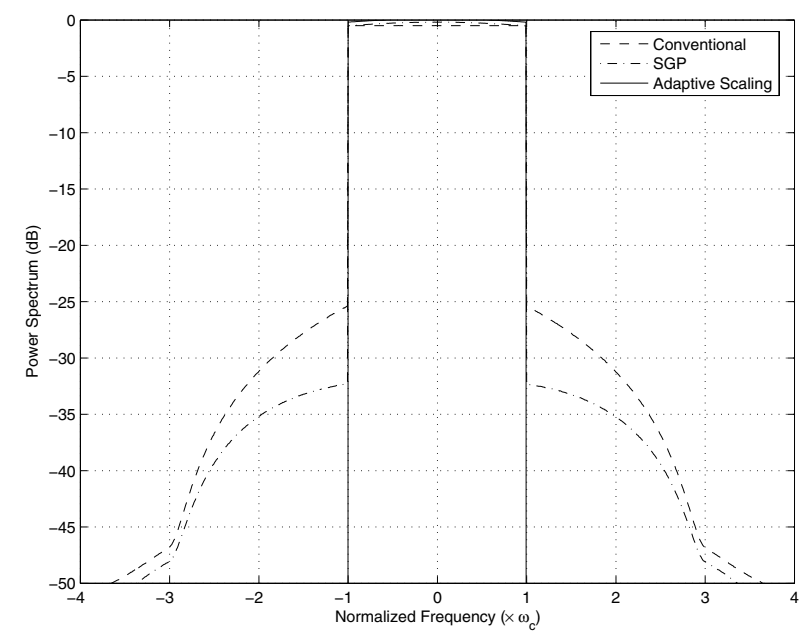

Fig. 3. Power spectrum comparison of the adaptive-scaling algorithm and SGP, where $L=3$ and $C=4.86 \mathrm{~dB}$.

Fig. 4 compares the BERs of the adaptive-scaling algorithm and SGP over an Additive White Gaussian Noise (AWGN) channel. The BER of the ideal OFDM system, where the HPA has a linear range, is shown in this figure as the dashed curve. The BER of the conventional OFDM with the SSPA (Conv. with SSPA) is also shown as the dotted curve. Although reducing the out-of-band radiation, the SGP algorithm is $0.4 \mathrm{~dB}$ worse than Conv. with SSPA at the BER of $10^{-5}$. On the other hand, the adaptive-scaling algorithm is only $0.1 \mathrm{~dB}$ worse than Conv. with SSPA, while entirely eliminating outof-band radiation.

\section{CONCLUSIONS}

In this paper, we have proposed an adaptive-scaling algorithm for the implementation of ACE. This algorithm is based on the clipping and filtering technique, and uses only the peak

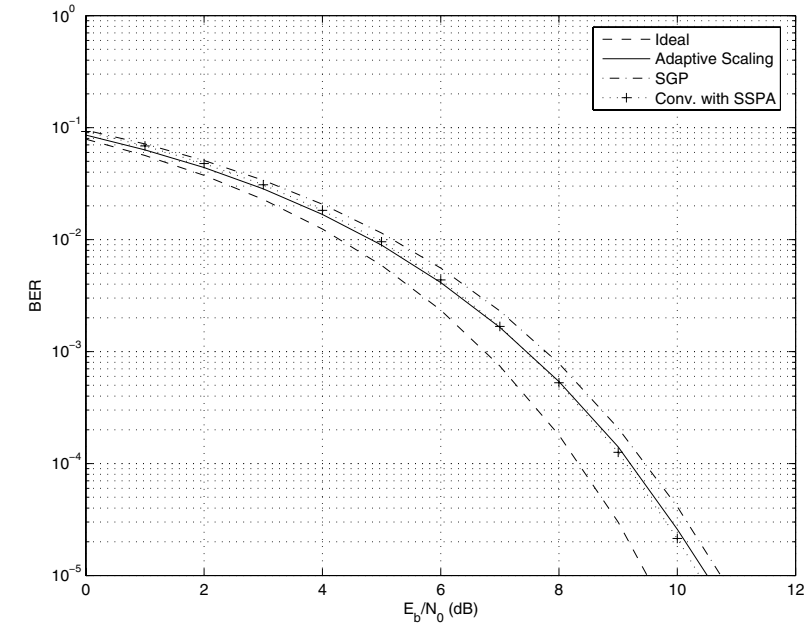

Fig. 4. BER comparison of the adaptive-scaling algorithm and SGP over the AWGN channel, where $L=3$ and $C=4.86 \mathrm{~dB}$.

samples of clipping noise to obtain optimum PAR reduction with a marginal average power increase. Simulation results show that the proposed algorithm outperforms the SGP in terms of PAR reduction, BER, and complexity.

\section{REFERENCES}

[1] R. van Nee and R. Prasad, OFDM for Wireless Multimedia Communications. Boston: Artech House Publishers, March 2000.

[2] S. H. Müller and J. B. Huber, "A novel peak power reduction scheme for OFDM," in 8th IEEE PIMRC (PIMRC'97), vol. 3, Helsinki, Sept. 1-4, 1997, pp. 1090-1094.

[3] C. Tellambura and A. Jayalath, "PAR reduction of an OFDM signal using partial transmit sequences," in 54th IEEE Veh. Technol. Conf. (VTC 2001 Fall), vol. 1, Atlantic City, NJ USA, 2001, pp. 465-469.

[4] C. Tellambura, "Improved phase factor computation for the PAR reduction of an OFDM signal using PTS," IEEE Commun. Lett., vol. 5, no. 4 , pp. 135-137, Apr. 2001.

[5] J. Davis and J. Jedwab, "Peak-to-mean power control in OFDM, Golay complementary sequences, and Reed-Muller codes," IEEE Trans. Inform. Theory, vol. 45, no. 7, pp. 2397-2417, Nov. 1999.

[6] X. Li and L. J. Cimini, "Effects of clipping and filtering on the performance of OFDM," IEEE Commun. Lett., vol. 2, no. 5, pp. 131133, May 1998.

[7] A. Gatherer and M. Polley, "Controlling clipping probability in DMT transmission," in 31st Asilomar Conf. on Signals, Systems and Computers, vol. 1, Pacific Grove, CA, Nov. 2-5, 1997, pp. 578-584.

[8] S. Leung, S. Ju, and G. Bi, "Algorithm for repeated clipping and filtering in peak-to-average power reduction for OFDM," IEE Elect. Lett., vol. 38, no. 25, pp. 1726-1727, Dec. 2002.

[9] J. Armstrong, "Peak-to-average power reduction for OFDM by repeated clipping and frequency domain filtering," IEE Elect. Lett., vol. 38, no. 5 , pp. 246-247, Feb. 2002.

[10] L. Wang and C. Tellambura, "A simplified clipping and filtering technique for PAR reduction in OFDM systems," IEEE Signal Processing Lett., vol. 12, no. 6, pp. 453-456, June 2005.

[11] — "Analysis of tone reservation technique for PAR reduction in OFDM systems," In preparation.

[12] J. Tellado, "Peak to average power reduction for multicarrier modulation," Ph.D. dissertation, Stanford University, Sept. 1999.

[13] B. Krongold and D. Jones, "An active-set approach for OFDM PAR reduction via tone reservation," IEEE Trans. Signal Processing, vol. 52, no. 2, pp. 495-509, Feb. 2004

[14] - "PAR reduction in OFDM via active constellation extension," IEEE Trans. Broadcast., vol. 49, no. 3, pp. 258-268, Sept. 2003. 